\title{
Substantial creep in healing human Achilles tendons. A pilot study
}

\author{
Per Aspenberg \\ Thorsten Schepull
}

Orthopedics, Department of Clinical and Experimental Medicine, Linköping University, Linköping, Sweden

Corresponding author:

Per Aspenberg

Orthopedics, Department of Clinical and Experimental Medicine, Linköping University

Hälsouniversitetet, US

558185 Linköping, Sweden

E-mail: per.aspenberg@liu.se

\section{Summary}

Background: healing after rupture of the Achilles tendon can be described in terms of mechanical properties of the new-formed tissue, constituting the tendon callus. In previous human studies, the elastic modulus and the density remained almost constant during 3 months after mobilization started, and then improved up to one year. So far, time-dependent deformation of the healing human tendon has not been reported.

Methods: in a series of 16 patients, operated with Achilles tendon suture, we implanted tantalum beads into the tendon and measured the distance between them repeatedly during 3 min of constant loading, using an ordinary image intensifier. The patients unloaded their leg for $\mathbf{3 0} \mathrm{min}$ before the test. To avoid bias, all images were investigated in a randomized and blinded order.

Results: total strain during $3 \mathrm{~min}$ of constant loading at 7 weeks post injury amounted to $5 \%$, and at 19 weeks to $3 \%$. About half of the strain, after the loading was applied, occurred during the second and third min. Considerable strain also occurred just before loading, when the patient was told that a load would be applied, but before this was actually done.

Conclusion: the measurements were crude, and this study should be seen as a pilot. Still, viscoelastic properties seem to dominate the mechanical behavior the healing Achilles tendon from start of mobilization to 19 weeks, at least when tested after $30 \mathrm{~min}$ rest. This deserves further studies with more precise methods.
KEY WORDS: Achilles tendon rupture, visco-elasticity, tendon healing.

\section{Introduction}

The early tendon regenerate after an Achilles tendon rupture is a watery tissue, likely to show time-dependent deformation during load. To our knowledge the extent of such deformation has not previously been estimated in humans. Estimations of mechanical properties of intact tendons have usually applied ultrasonography ${ }^{1}$. This method measures the entire length of the tendon, and is therefore unlikely to capture the properties of the injured region. Methods are currently under development that probably will enable localized measurements, based on elastography. Using this method, the viscous properties of intact tendons have recently been described for the first time ${ }^{2}$, but so far there is not much known about the mechanics of healing human Achilles tendons.

We have previously used tantalum beads implanted into the tendon during surgery for Achilles tendon rupture. This allowed use of the radiosterometric analysis (RSA) technique ${ }^{3}$, which can measure the distance between the beads with a precision around $0.1 \mathrm{~mm}$. Strain during loading will increase the distance between the beads. By measuring tendon strain using two different weights for loading, we achieved 2 points of the load-deformation curve. Assuming a purely elastic behavior, we then estimated what we thought to be tendon stiffness and modulus of elasticity. These measurements were sufficiently accurate to allow prognostic statements ${ }^{3-6}$ and allowed us to show a statistically significant improvement due to early controlled loading in a randomized trial $^{4}$. The estimated modulus seemed to remain constant from 7 to 19 weeks after injury, and then increased to 52 weeks after injury. Similarly, tissue density remained unchanged until 19 weeks and then increased dramatically to 52 weeks ${ }^{7}$. In contrast, the cross-sectional area increased from 7 to 19 weeks and then receded. Together, these data seem to suggest that tissue properties do not really change during the regenerative phase of healing, and that remodeling is not sufficiently effective to influence tissue characteristics until after 19 weeks.

Our previous studies have assumed the tissue to be mainly elastic and disregarded the viscous component of deformation. By using pre-loading and standardized time points, a time-dependent component of 
deformation has been supposed to be similar across groups, enabling group comparisons to be valid. Still, the estimated Young's modulus could have a systematic error.

We here describe the time-dependent strain during constant loading in a series of 16 patients.

\section{Methods}

This study is regarded as a pilot, to find if time-dependent strain could be measured at all with the methods available. 16 patients (age 24-57 years; 3 women) with acute Achilles tendon ruptures volunteered to participate after written information and consent. The study was approved by the Regional Ethics Board in Linköping.

\section{Surgery and after-treatment}

Surgery was performed using a dorso-medial approach and an open technique. All patients were operated on in local anaesthesia, using mepivacaine hydrochloride (Carbocaine) with adrenaline. The proximal and distal tendon stubs were adapted using a resorbable suture (Vicryl ${ }^{\circledR}$ Size 0 ) and using a single loop, modified Kessler technique. During surgery, 4 Tantalum metal markers, with a diameter of $0.8 \mathrm{~mm}$, were placed within the tendon substance. Two markers were placed in the proximal part of the tendon and two were placed in the distal part (Fig. 1). The paratenon was closed thoroughly and the skin was sutured with a resorbable intracutaneous suture (Monocryl 3-0).

After surgery, a short leg cast was applied, with the foot in equinus position. The cast was removed after

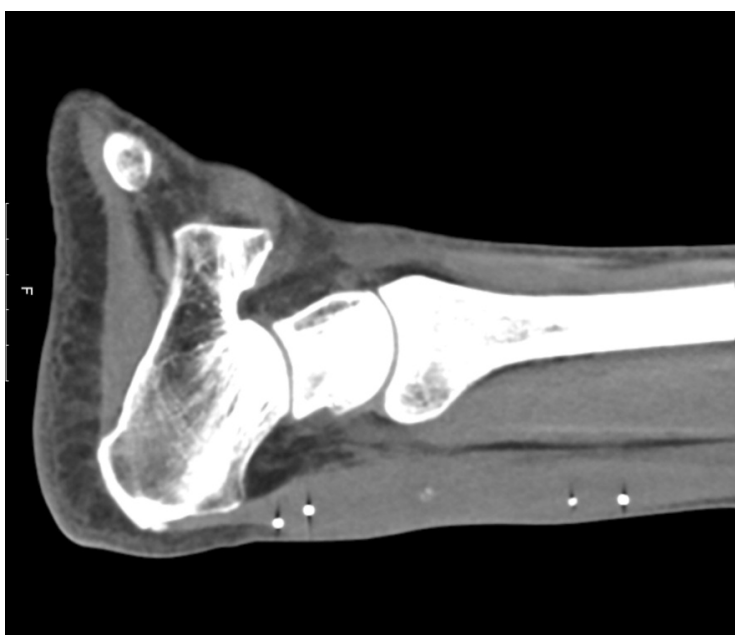

Figure 1. CT, showing the tendon callus with implanted Tantalum beads. Each patient received 2 beads proximal and 2 distal to the rupture. Only the beads closest to the injury were used. The beads are here enlatrged by CT artefacts.
2 weeks and replaced by a removable foam walker boot for another 5 weeks. Patients were provided with three heel wedges in the beginning, and removed one heel wedge every week, i.e. the foot was in neutral position without any heel wedge after 5 weeks. After 7 weeks in total ( 5 weeks in a foam walker), the walker was removed. Full weight-bearing was allowed as tolerated from the beginning. For the two first weeks, as long as the patients had a cast in an equinus position, they could only bear weight on the frontal part of the cast.

During the period with the foam walker, patients were instructed to remove the brace twice a day and perform unloaded ankle motion exercises. Most of the patients also underwent measurements of ankle moment during gait wearing a brace. That study is reported separately ${ }^{8}$.

\section{Preloading before strain measurement}

Before the examination, the patient was asked to perform 15 seconds of loading against a $15 \mathrm{~kg}$ weight as described below. This was done twice. Thereafter, the patient did not bear weight on the injured leg for 30 minutes, before the measurement of time-dependent strain, which was performed as follows.

\section{Strain measurement}

The patients sat on an X-ray examination table with the foot in a specially designed frame and with 8 degrees of plantar flexion (Fig. 2). The frame allowed us to apply a pedal to the forefoot. The pedal was connected via strings to weights, to produce the desired loading. The pedal pivoted around an axis with an adjustable distance from the posterior aspect of the heel, which allowed estimation of the moment arms from radiographs. The patients were then asked to keep the foot in position and to resist the dorsalflexion moment derived from the pedal during loading. Thereafter the weights were applied. Images capturing the tantalum beads were taken with an image intensifier. First, one single image was tak-

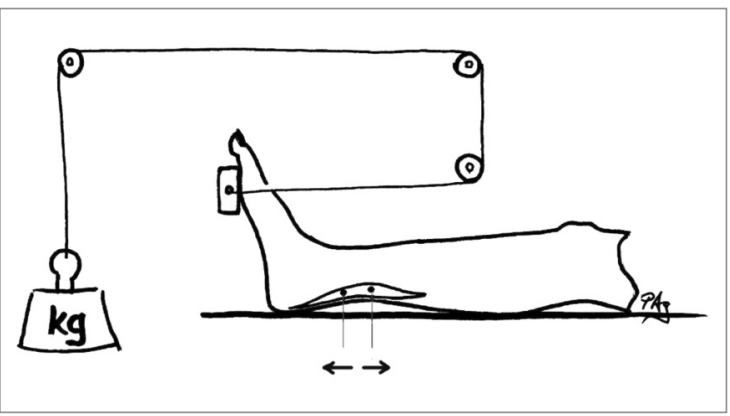

Figure 2. Principal sketch of the loading apparatus. The tendon callus and the implanted beads are shown. Small arrows indicate the elongation that was measured over time. 
en before loading (Fig. 3). Thereafter the machine took one image per second automatically. The weight was applied about 5 seconds after the machine started, and the machine stopped 5 seconds after the weight was applied. The weight was applied with some care, but the increase to full load occurred during less than a second. Images were also taken 1, 2 and 3 minutes after loading started. Thereafter the weight was removed.

The distances between the distal and proximal tantalum beads were measured on the images using a conventional system for measurements on digital radiographs (SECTRA IDS7). In order to avoid bias, the measurements for each patient were made with the images in random order, without the investigator knowing where in the sequence of events the image was taken.

\section{Statistics}

The hypothesis was that a measurable increase in strain would occur after the instantaneous (elastic) deformation. Because the time point for instantaneous deformation was sometimes hard to define, we chose to refine the hypothesis to strain during the second and third minutes. This was tested with Wilcoxon's signed rank test.

\section{Results}

\section{Hypothesis test}

There was a significant increase in strain from 1 minute after loading was applied to 3 minutes ( $p=0.025$ for 7 weeks and $p=0.002$ for 19 weeks).

\section{Descriptive}

At 7 weeks, all patients could do the test with $10 \mathrm{~kg}$ loading of the pedal, except 2 patients, who could only do it with $5 \mathrm{~kg}$. At 19 weeks, all could easily resist $15 \mathrm{~kg}$. The data for 4 patients at 7 weeks disappeared in a computer crash.

A considerable strain was noted immediately after the patients were informed that loading would be applied, but before this was actually done (Figs. 4, 5). This indicates that the patients braced themselves to resist the test load by isometric contraction. Strain due to this contraction at 7 weeks was median $0.73 \%$ (interquartile range 0.27 to 2.6 ; range 0 to 5.0 ) and at 19 weeks it was $0.19 \%$ (interquartile range -0.43 to 0.5 ; range -0.43 to 1.4$)(p=0.06)$. The curves for the first seconds at 7 weeks show no clear point where loading was applied, suggesting that no clear instantaneous strain occurred. At 19 weeks, most tendon
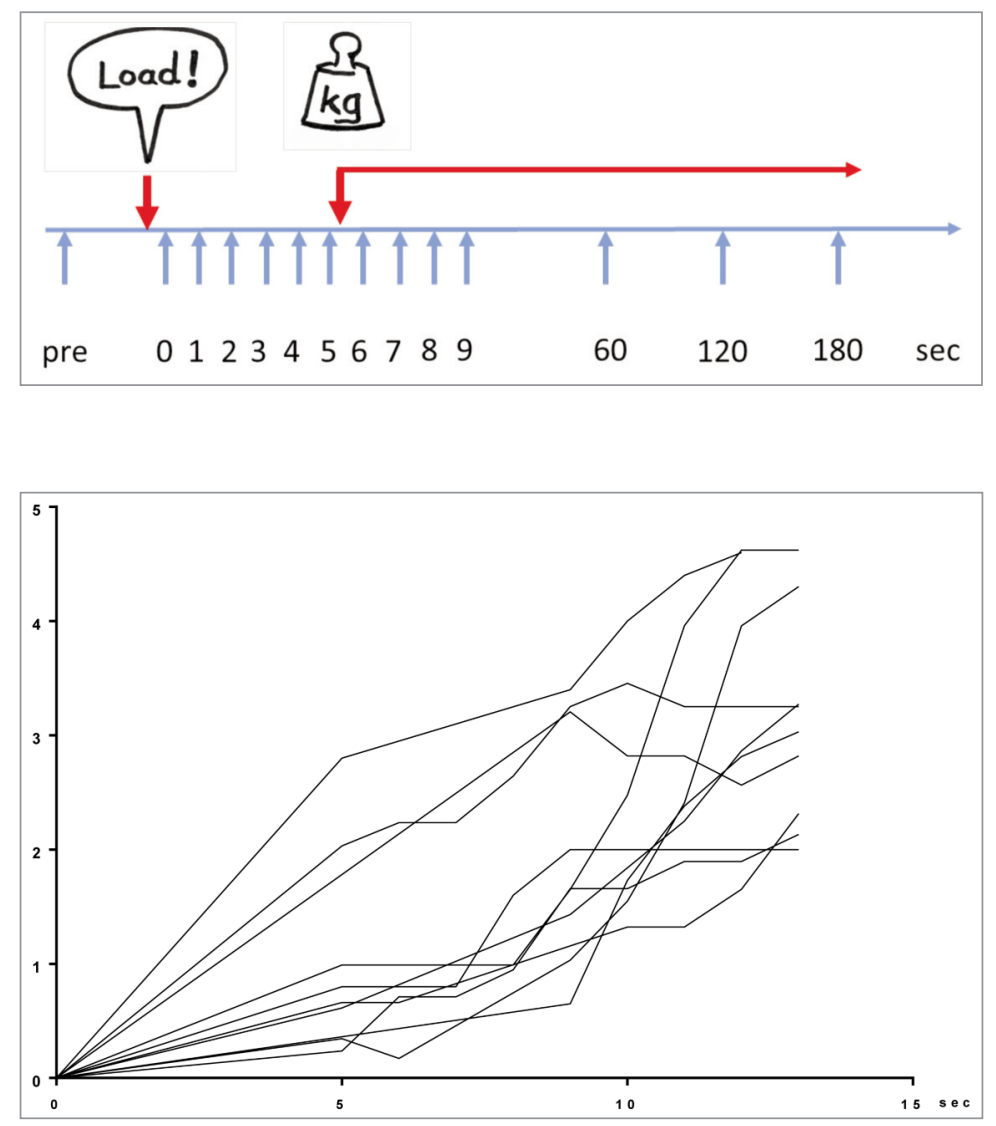

Figure 3. Time schedule for each examination. Time for imaging is marked with blue arrows. After a pre-examination (pre) the patients were warned that loading would be applied (!), and images were taken at $1 \mathrm{sec}$ intervals. After $5 \mathrm{sec}$, the load $(\mathrm{kg})$ was actually applied.

Figure $4 \mathrm{~A}$. Deformation curves for patients at 7 weeks after Achilles tendon rupture. Vertical axis denotes percent strain. Time 0 refers to immediately before the patient was warned that loading would be applied. Time 5 sec marks when the machine started to take 1 image per second. Loading was applied at about time $10 \mathrm{sec}$. Note that no obvious instantaneous deformation can be seen. 

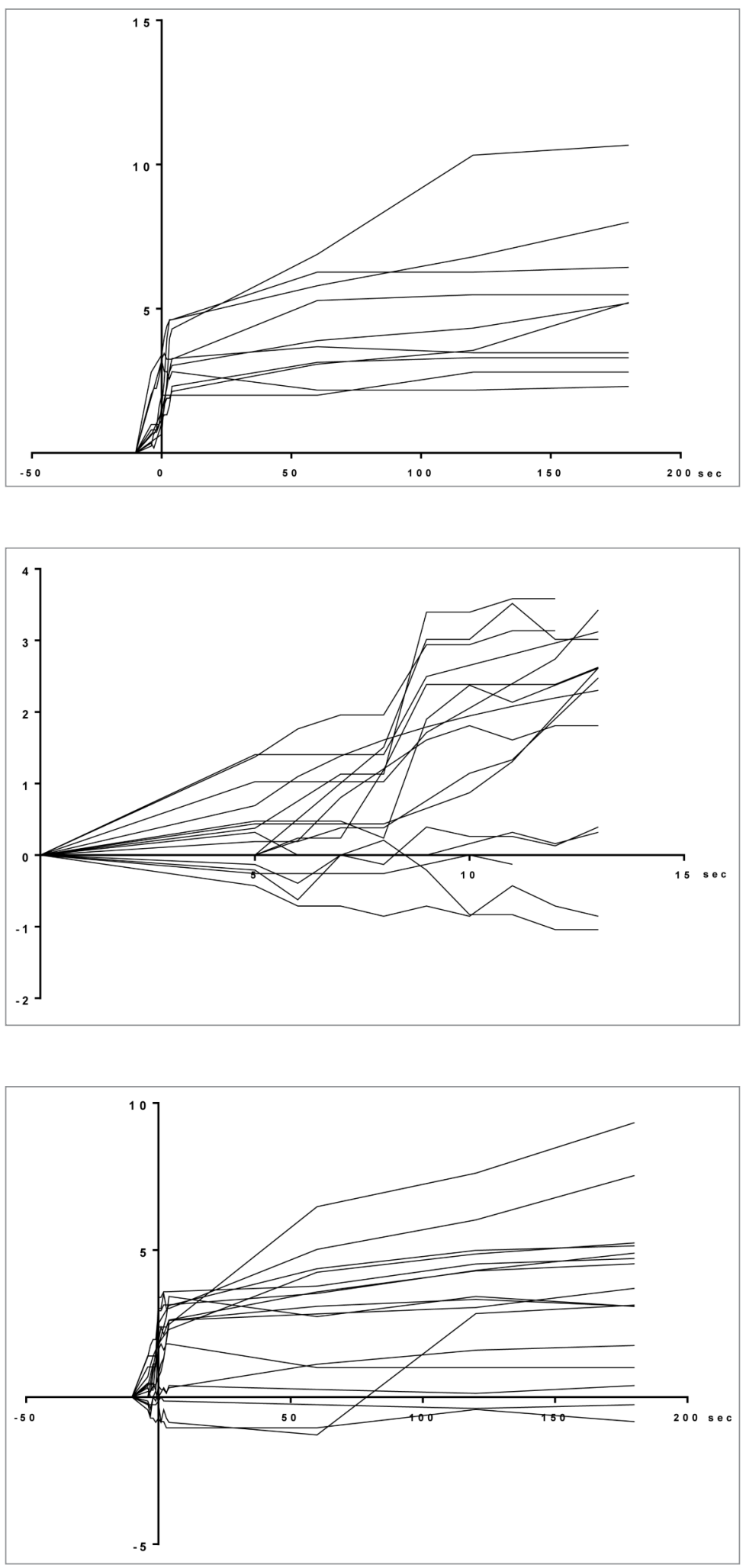

Figure $4 \mathrm{~B}$. Similar curve as Figure $4 \mathrm{~A}$, but here for the entire 3 minutes. Time 0 marks when loading was applied.
Figure $5 \mathrm{~A}$. Deformation curves for patients at 19 weeks after Achilles tendon rupture. Vertical axis denotes percent strain. Time 0 refers to immediately before the patient was warned that loading would be applied. Time 5 sec marks when the machine started to take 1 image per second. Loading was applied at about time $10 \mathrm{sec}$. Note that a sudden increase in strain is seen for most patients, indicating that instantaneous deformation occurred when loading was applied.
Figure 5 B. Similar curve as Figure 5 A, but here for the entire 3 minutes. Time 0 marks when loading was applied. showed such an instantaneous strain, suggesting that the tendon had now achieved some elastic properties. In all patients, the tendon became longer between 1 and 3 minutes after loading (except one patient at 7 weeks). The median increase in strain during the second and third minutes of loading at 7 weeks after injury, expressed as percent units, was $0.5 \%$ (interquartile range 0.12 to 2.1 ; range -0.20 to 3.8 ). After 19 
weeks it was $0.77 \%$ (interquartile range 0.14 to 1.4 ; range 0 to 4.4). This strain amounted to about half of the total strain from the time loading started.

Total strain at 7 weeks was $5.2 \%$ (interquartile range 3.3 to 6.4 ; range 2.3 to 11 ). At 19 weeks it was $3.1 \%$ (interquartile range 1.1 to 4.9 ; range -0.83 to 9.3 ). Thus, both the deformation occurring immediately before start of loading, during the first second of loading, and during the following 3 minutes all contribute considerably to total deformation.

\section{Discussion}

This is mainly a descriptive paper ${ }^{9}$. The radiation doses associated with conventional RSA measurements are too large for more than a few images per patient, hence the cruder, low radiation method in this paper. Still, we were able to show a statistically significant increase in tendon callus length during the second and third minutes of constant loading, which amounted to about half of the increase after loading started. The variation was large, but because the radiographs were measured in random order, and still showed a consistent pattern in most cases, the variation can't be explained only by measuring error. We would be happy to see our measurements confirmed, using more hightech techniques that are not available to us.

One consistent finding from over 100 patients tested with RSA was that estimated modulus of elasticity at 7 weeks correlated well with the ability to do one-legged heel-raises (normalized to the uninjured side) at 1 year after the injury. Pooled data from 3 randomized trials ${ }^{4-6}$ show that $32 \%$ of the variance in heel-raise index can be explained by the estimated modulus of elasticity at 7 weeks ( $p<0.0001$, pooled data not shown). This confirms that measurements according to the used RSA protocol contains meaningful information, in spite of the assumption of purely elastic behavior. However, the connection between early callus mechanics and late muscle performance has been hard to explain. The current unexpected finding, that strain occurred before the load was actually applied, might provide a possible, hypothetical explanation, as follows.

The patients contracted their calf muscles before the load was applied. The pre-loading strain might possibly be linked to psychology, or attitude to physical activity. A patient who is confident that he or his Achilles tendon can deal with the loading might more easily brace himself, and thus pre-stress his tendon callus more. During the RSA examinations of our previous studies, this might have added to the measured length of the tendon during the first, low-load loading, making it more similar to the ensuing high-load loading. With such pre-stress the recorded values will, incorrectly, indicate a higher stiffness. The same confident patient might also perform better at one year, because his muscle function is again related to his attitude to physical activity: he has trained more. There- fore, the enigmatic link between early tendon callus stiffness and late muscle function might perhaps to some extent be related to patient personality.

Our RSA studies used $15 \mathrm{~s}$ of preloading with full load. A lasting elongation from that preload of $0.8 \%$ has previously been described, and it remained similar from 7 weeks (brace removal) to 19 weeks $^{3}$. The deformation during the second and third minutes in the current study was also similar between those time points. This concurs with stiffness measurements, and also with measurements of radiodensity, all indicating that the material properties of the callus are similar at these time points. It seems that the healing process simply adds more and more material of the same quality during the first months. Later, during the second half year, the quality improves dramatically ${ }^{3-6}$. We did not try to measure plastic deformation at 1 year, mainly because we know from RSA measurements that the tendon is stiffer, suggesting that image intensifiers would not provide sufficient precision.

In conclusion, there is considerable visco-elastic deformation during the first loading after rest, 7 and 19 weeks after Achilles tendon rupture. Our previous estimates of Young's modulus should be interpreted with caution.

\section{References}

1. Agres AN, Duda GN, Gehlen TJ, et al. Increased unilateral tendon stiffness and its effect on gait 2-6 years after Achilles tendon rupture. Scandinavian journal of medicine \& science in sports. 2015

2. Suydam SM, Soulas EM, Elliott DM, et al. Viscoelastic properties of healthy achilles tendon are independent of isometric plantar flexion strength and cross-sectional area. J Orthop Res. 2015;33(6):926-931.

3. Schepull T, Kvist J, Andersson C, et al. Mechanical properties during healing of Achilles tendon ruptures to predict final outcome: a pilot Roentgen stereophotogrammetric analysis in 10 patients. BMC musculoskeletal disorders. 2007;8:116.

4. Schepull T, Aspenberg P. Early controlled tension improves the material properties of healing human achilles tendons after ruptures: a randomized trial. The American journal of sports medicine. 2013;41(11):2550-2557.

5. Schepull T, Kvist J, Aspenberg P. Early E-modulus of healing Achilles tendons correlates with late function: similar results with or without surgery. Scandinavian journal of medicine \& science in sports. 2012;22(1):18-23.

6. Schepull T, Kvist J, Norrman H, et al. Autologous platelets have no effect on the healing of human achilles tendon ruptures: a randomized single-blind study. The American journal of sports medicine. 2011;39(1):38-47.

7. Schepull T, Aspenberg P. Healing of human Achilles tendon ruptures: radiodensity reflects mechanical properties. Knee surgery, sports traumatology, arthroscopy: official journal of the ESSKA. 2013.

8. Sandberg $\mathrm{O}$, Dånmark I, Eliassona $\mathrm{P}$, et al. Influence of a lower leg brace on traction force in healthy and ruptured Achilles tendons. Muscle Ligament Tendon J. 2015;5(2):63-67.

9. Padulo J, Oliva F, Frizziero A, et al. Muscle, Ligaments and Tendons Journal. Basic principles and recommendations in clinical and field science research. MLTJ. 2013;4:250-252. 